PROCEEDINGS OF THE

AMERICAN MATHEMATICAL SOCIETY

Volume 132, Number 9, Pages 2543-2547

S 0002-9939(04)07369-1

Article electronically published on March 25, 2004

\title{
THE EXCHANGE PROPERTY FOR PURELY INFINITE SIMPLE RINGS
}

PERE ARA

(Communicated by Martin Lorenz)

\begin{abstract}
It is proven that every purely infinite simple ring is an exchange ring. This result is applied to determine those Leavitt algebras that are exchange rings.
\end{abstract}

\section{INTRODUCTION}

Purely infinite simple $C^{*}$-algebras, introduced by J. Cuntz in 1981 [11, have played a central role in the development of the theory of $C^{*}$-algebras in the last two decades. One of the most important advances in the program of classifying separable $C^{*}$-algebras through $K$-theory, proposed by Elliott in the early seventies, was obtained in this context by Kirchberg [14] and Phillips [17, who showed that nuclear separable unital purely infinite simple $C^{*}$-algebras are classified by $K$-theoretic invariants. See [19] for a survey on the classification of nuclear $C^{*}$ algebras.

Recall that a $C^{*}$-algebra $A$ has real rank zero if every selfadjoint element in $A$ is a norm-limit of a sequence of selfadjoint invertible elements, see [8]. A fundamental result is that every purely infinite simple $C^{*}$-algebra has real rank zero. This was shown by Zhang in [21] and also by Brown and Pedersen in [8].

A suitable notion of purely infinite simple ring was introduced by Goodearl, Pardo and the author in [4. A simple unital ring $R$ is purely infinite in case it is not a division ring and for each nonzero element $x$ in $R$, there are elements $z, t$ in $R$ such that $1=z x t$. (The rings satisfying the latter property have been termed 1-simple rings by P. M. Cohn [10.) Since the $C^{*}$-algebras of real rank zero are exactly the ones that are exchange rings 2, Theorem 7.2], the fundamental result established by Zhang and Brown and Pedersen is equivalent to the statement that every purely infinite simple $C^{*}$-algebra is an exchange ring. (The definition of exchange ring is given at the beginning of Section 1.) This motivated the following question, posed in [4, 1.8(a)]: Is every purely infinite simple ring an exchange ring? In this paper, we answer this question in the affirmative. In fact, the proof we

Received by the editors March 15, 2003 and, in revised form, May 30, 2003.

2000 Mathematics Subject Classification. Primary 16E50, 16 D30.

Key words and phrases. Purely infinite simple ring, exchange ring, Leavitt algebra.

The author was partially supported by MEC-DGESIC, and by the Comissionat per Universitats i Recerca de la Generalitat de Catalunya. 
have found is quite simple, thus providing an elegant algebraic generalization of the fundamental $C^{*}$-algebra result mentioned above. The existing proofs of the fact that every purely infinite simple $C^{*}$-algebra has real rank zero are of a topological nature, and cannot be adapted to cover the result presented here.

We will also show a corresponding result for non-unital rings. The exchange property for a purely infinite simple ideal of a unital ring was a technical hypothesis that needed to be added to get some of the results on $Q B$-rings obtained in 6 ; see for example [6] Theorem 1.12, Proposition 3.2]. It follows from Corollary 1.2 that this hypothesis is automatically satisfied. The reader is referred to [5] for background material on $Q B$-rings.

We will use our result to determine which Leavitt algebras are exchange rings. These algebras were defined and studied by Leavitt in [15] and [16], and their ringtheoretical properties have been investigated by several authors, among them Cohn, Skornyakov and Bergman, see e.g. [9, 2.11] and [7].

\section{Purely infinite Rings}

Following Warfield [20], we say that a unital ring $R$ is an exchange ring in case the regular left $R$-module ${ }_{R} R$ satisfies the (finite) exchange property, that is, for every $R$-module $A$ and any decompositions

$$
A=M^{\prime} \oplus N=\bigoplus_{i=1}^{n} A_{i}
$$

with $M^{\prime} \cong{ }_{R} R$, there exist submodules $A_{i}^{\prime} \subseteq A_{i}$ such that

$$
A=M^{\prime} \oplus\left(\bigoplus_{i=1}^{n} A_{i}^{\prime}\right)
$$

By a result obtained independently by Goodearl [13 and Nicholson [18], $R$ is an exchange ring if and only if for every element $a$ in $R$ there is an idempotent $e$ in $R$ such that $e \in R a$ and $1-e \in R(1-a)$.

If $R$ is a ring and $e, f \in R$ are idempotents, we say that $e$ and $f$ are (Murray-von Neumann) equivalent (denoted $e \sim f$ ) provided that there exist elements $x \in e R f$ and $y \in f R e$ such that $x y=e$ and $y x=f$. This is equivalent to demanding that $e R \cong f R$ as right $R$-modules.

An idempotent $e$ in a ring $R$ is infinite if there exist orthogonal idempotents $f, g \in R$ such that $e=f+g$ while $e \sim f$ and $g \neq 0$. A simple unital ring $R$ is said to be purely infinite in case every nonzero left ideal contains an infinite idempotent. By [4, Theorem 1.6], a simple ring $R$ is purely infinite if and only if $R$ is not a division ring and for every nonzero element $a \in R$ there are $x, y \in R$ such that $x a y=1$. See [4 Section 1] for the elementary properties and basic examples of purely infinite simple rings.

Theorem 1.1. Every purely infinite simple unital ring is an exchange ring.

Proof. Let $R$ be a purely infinite simple ring. Let $a$ be an element in $R$ and set $b=1-a$. By [18, Proposition 1.1], it is enough to check that there is an idempotent $e$ such that $e \in R a$ and $R=R e+R b$. If $a=a^{2}$ there is nothing to prove. So we can assume that $R a \cap R b=R(a b) \neq 0$. By definition of purely infinite simple, 
there is a nonzero idempotent $g$ such that $g \in R a \cap R b$. By [4, Theorem 1.6], there are $x, y \in R$ such that $x g y=1$. Now consider the element

$$
e=g+g y a(1-g) \text {. }
$$

Clearly, $e$ is an idempotent, and since $g \in R a$ we have $e \in R a$. In order to prove that $R=R e+R b$, it is enough to show that $a \in R e+R b$. From the identity

$$
x e=x g+(x g y) a(1-g)=x g+a-a g
$$

we get $a=x e+(a-x) g \in R e+R g \subseteq R e+R b$. Therefore $R=R e+R b$, as desired. This completes the proof that $R$ is an exchange ring.

We now extend the above result to non-unital rings. The definition of purely infinite simple ring is the same as above: a simple ring $R$ is purely infinite in case every nonzero left ideal contains an infinite idempotent. If $x$ is a nonzero element in a purely infinite simple ring and $e=(e y) x$ is an infinite idempotent in the left ideal generated by $x$, then $f=x(e y)$ is an infinite idempotent in the right ideal generated by $x$, showing that the notion is left-right symmetric.

The concept of exchange ring was generalized to the non-unital case in [1]. Namely, a ring $R$ is an exchange ring when for every element $a$ in $R$ there are an idempotent $e$ in $R$ and elements $r, s$ in $R$ such that $e=r a=a+s-s a$. Note that this reduces to the Goodearl-Nicholson characterization of exchange rings in case $R$ is a unital ring.

We can now extend Theorem 1.1 to the non-unital case.

Corollary 1.2. Let $R$ be a purely infinite simple non-unital ring. Then $R$ is an exchange ring.

Proof. Take a nonzero idempotent $p$ in $R$, and consider the corner ring $S=p R p$. It is easy to check that $S$ is a purely infinite simple unital ring. It follows from Theorem 1.1 that $S$ is an exchange ring.

To show that $R$ is an exchange ring, we use the theory of Morita equivalence for rings without identity; see, for example, [12].

Let us denote by $R^{2}$ the set of all finite sums of products of two elements in $R$. Clearly $R^{2}$ is a two-sided ideal of $R$, and $R^{2} \neq 0$ because $R$ contains nonzero idempotents. Since $R$ is a simple ring, we get $R=R^{2}$. Therefore $R$ is an idempotent ring, and there is a Morita context $(R, S, R p, p R)$, showing that $R$ and $S$ are Morita equivalent. Since $S$ is an exchange ring, it follows from [3, Theorem 2.3] that $R$ is an exchange ring.

\section{An APPLiCATion}

In this section, we apply the main result of this note to determine the Leavitt algebras that are exchange rings.

Leavitt algebras are universal examples of non-IBN algebras. For any field $k$, and for any two natural numbers $m, n$, we denote by $V_{m, n}$ the $k$-algebra with a universal isomorphism $i: n V_{m, n} \longrightarrow m V_{m, n}$, and we denote by $U_{m, n}$ the $k$-algebra with a universal pair of morphisms $i: n U_{m, n} \longrightarrow m U_{m, n}$ and $j: m U_{m, n} \longrightarrow n U_{m, n}$ such that $j i=1_{n U_{m, n}}$. Leavitt, Cohn, Skornyakov and Bergman proved some fundamental properties of these algebras. In particular, the monoid of isomorphism classes of finitely generated projective modules of these examples was computed by Bergman in [7]. 
Theorem 2.1. Let $k$ be a field, let $m, n$ be positive integers with $m \leq n$, and let $U_{m, n}$ and $V_{m, n}$ be the Leavitt $k$-algebras defined above. Then the following conditions are equivalent:

(a) $U_{m, n}$ is an exchange ring;

(b) $V_{m, n}$ is an exchange ring;

(c) $m=1$ and $n>1$.

Proof. The $k$-algebra $U_{m, n}$ has a system of $2 n m$ generators $x_{i j}, y_{j i}, i=1, \ldots, n$, $j=1, \ldots, m$, with defining relations which in matrix form can be written

$$
X Y=I_{n}, \quad X=\left(x_{i j}\right), \quad Y=\left(y_{j i}\right) .
$$

The algebra $V_{m, n}$ is the factor ring of $U_{m, n}$ modulo the ideal generated by the relations

$$
Y X=I_{m}
$$

(a) $\Longrightarrow(b)$. This follows from the fact that a factor ring of an exchange ring is also an exchange ring.

(b) $\Longrightarrow$ (c). It is well known (and easy to prove) that an integral domain $R$ is an exchange ring if and only if it is a local ring. Here we are using the standard definition for noncommutative local rings as the rings for which the non-units form a proper two-sided ideal.

If $m=n=1$, then $V_{1,1}=k\left[t, t^{-1}\right]$, a Laurent polynomial ring. So $V_{1,1}$ is an integral domain, but it is not a local ring. It follows that $V_{1,1}$ is not an exchange ring.

If $m>1$, then $V_{m, n}$ is an $(m-1)$-fir by 7 , Theorem 6.1]. In particular $V_{m, n}$ is an integral domain. It is easy to see that the only invertible elements in $V_{m, n}$ are the nonzero elements of $k$; cf. [16, proof of Theorem 9]. Therefore, $V_{m, n}$ is an integral domain that is not a local ring, and so it is not an exchange ring.

(c) $\Longrightarrow$ (a). Assume $n>1$. By [4, Theorem 4.2], the algebra $V_{1, n}$ is a purely infinite simple ring. It follows from Theorem 1.1 that $V_{1, n}$ is an exchange ring. Now $U_{1, n}$ has a unique maximal ideal $M$, which is the ideal generated by the idempotent

$e:=1-\sum_{i=1}^{n} y_{i} x_{i}$. The ideal $M$ coincides with the socle of $U_{1, n}$, and it is a von Neumann regular ideal. In fact, it is easy to check that $M$ is linearly spanned by an infinite family of matrix units; cf. [4, Section 4].

Thus we have proved that $U_{1, n}$ has a von Neumann regular ideal $M$ such that the factor ring $U_{1, n} / M \cong V_{1, n}$ is an exchange ring. It follows from [1, Corollary $2.4]$ that $U_{1, n}$ is also an exchange ring.

\section{REFERENCES}

[1] P. Ara, Extensions of exchange rings, J. Algebra 197 (1997), 409-423. MR 98j:16021

[2] P. Ara, K. R. Goodearl, K. C. O'Meara, and E. Pardo, Separative cancellation for projective modules over exchange rings, Israel J. Math. 105 (1998), 105-137. MR 99g:16006

[3] P. Ara, M. Gómez Lozano, and M. Siles Molina, Local rings of exchange rings, Comm. in Algebra 26(12) (1998), 4191-4205. MR 99h:16036

[4] P. Ara, K. R. Goodearl, and E. Pardo, $K_{0}$ of purely infinite simple regular rings, $K$-Theory 26 (2002), 69-100.

[5] P. Ara, G. K. Pedersen, and F. Perera, An infinite analogue of rings with stable rank one, J. Algebra 230 (2000), 608-655. MR 2001g:16018

[6] P. Ara, G. K. Pedersen, and F. Perera, Extensions and pullbacks in QB-rings, Algebras and Representation Theory, to appear. Available at ArXiv: math.RA/0107104.

[7] G. M. Bergman, Coproducts and some universal ring constructions, Trans. Amer. Math. Soc. 200 (1974), 33-88. MR 50:9971 
[8] L. G. Brown and G. K. Pedersen, $C^{*}$-algebras of real rank zero, J. Funct. Anal. 99 (1991), 131-149. MR 92m:46086

[9] P. M. Cohn, Free Rings and Their Relations, Second Edition, London Mathematical Society Monographs 19, Academic Press, London, 1985. MR 87e:16006

[10] P. M. Cohn, On n-simple rings, preprint.

[11] J. Cuntz, K-theory for certain $C^{*}$-algebras, Annals of Math. 113 (1981), 181-197. MR 84c: 46058

[12] J. L. García and J. J. Simón, Morita equivalence for idempotent rings, J. Pure and Applied Algebra 76 (1991), 39-56. MR 93b:16010

[13] K. R. Goodearl and R. B. Warfield, Jr., Algebras over zero-dimensional rings, Math. Annalen 223 (1976), 157-168. MR 54:357

[14] E. Kirchberg, The classification of purely infinite $C^{*}$-algebras using Kasparov's theory, Fields Institute Comm. Series, 2001 (to appear).

[15] W. G. Leavitt, Modules without invariant basis number, Proc. Amer. Math. Soc. 8 (1957), 322-328. MR 18:789a

[16] W. G. Leavitt, The module type of a ring, Trans. Amer. Math. Soc. 103 (1962), 113-130. MR 24:A2600

[17] N. C. Phillips, A classification theorem for nuclear purely infinite simple $C^{*}$-algebras, Documenta Math. 5 (2000), 49-114. MR 2001d:46086b

[18] W. K. Nicholson, Lifting idempotents and exchange rings, Trans. Amer. Math. Soc. 229 (1977), 269-278. MR 55:12757

[19] M. Rørdam and E. Størmer, Classification of nuclear $C^{*}$-algebras. Entropy in Operator Algebras, Encyclopedia of Mathematical Sciences, Operator Algebras and Non-Commutative Geometry, Springer-Verlag, Berlin, Heidelberg, New York, 2002. MR 2002i:46047

[20] R. B. Warfield, Jr., Exchange rings and decompositions of modules, Math. Ann. 199 (1972), 31-36. MR 48:11218

[21] S. Zhang, A property of purely infinite simple $C^{*}$-algebras, Proc. Amer. Math. Soc. 109 (1990), 717-720. MR 90k:46134

Departament de Matemàtiques, Universitat Autònoma de Barcelona, 08193, Bellaterra (BArcelona), Spain

E-mail address: para@mat.uab.es 